\title{
Elevated risk of death persists beyond 30 days after mitral valve surgery
}

\author{
Makoto Mori, MD, Sameh Yousef, MD, Matthew Pichert, MD, Thais Faggion Vinholo, BS, MSc, and \\ Arnar Geirsson, MD, New Haven, Conn
}

\author{
From the Section of Cardiac Surgery, Yale University School of Medicine, New Haven, Conn. \\ This study was not funded. \\ Disclosures: Authors have nothing to disclose with regard to commercial support. \\ Institutional Review Board protocol ID 2000020356, approval date March 2, 2018 \\ Received for publication April 24, 2019; revisions received June 26, 2019; accepted for publication July 20, 2019; \\ available ahead of print Aug 14, 2019 \\ Address for reprints: Arnar Geirsson, MD, Section of Cardiac Surgery, Yale School of Medicine, BB204, 330 Ce- \\ dar St, PO Box 208039, New Haven, CT 06510 (E-mail: arnar.geirsson@yale.edu). \\ J Thorac Cardiovasc Surg 2020;159:e171-3 \\ 0022-5223/\$0.00 \\ Published by Elsevier Inc. on behalf of The American Association for Thoracic Surgery \\ https://doi.org/10.1016/j.jtcvs.2019.07.021
}

\begin{abstract}
Although persistence of postoperative risk of death beyond 30 days has been demonstrated in coronary artery bypass grafting, ${ }^{1}$ this has not been investigated well in patients who underwent mitral valve (MV) surgeries. Increasing attention to outcomes beyond 30-days as a quality metric is evident through federally funded programs, such as the Hospital Readmission Reduction Program ${ }^{2}$ and the newly developed reimbursement scheme to incentivize coordination of postacute phase of care. ${ }^{3}$ To inform such initiatives for patients undergoing MV operations, we aimed to characterize temporal change in the risk of death within 1 year after MV repair and MV replacement (MVR).
\end{abstract}

\section{METHODS}

We conducted a retrospective cohort study on consecutive patients who underwent either concomitant or isolated MVR or MV repair at our center between 2011 and 2016. Twelve surgeon performed MVR and MV repair during the study period. In-hospital, 30, 90, 180, and 365-day mortalities and survival estimates were calculated for each cohort. Posthospital survival was adjudicated according to patient-level Connecticut State Vital Statistics. The Vital Statistics data do not capture those residing outside Connecticut, and this group was excluded. Kaplan-Meier analysis generated survival estimates, and the time-average of slope of the survival estimate was calculated at each time point to evaluate changes in the monthly risk of death during the 1 year following the surgery. Bivariate analyses were performed with the $\chi^{2}$ test or Fisher exact test for categorical variables and the Wilcoxon rank sum test for continuous variables. Analyses were conducted with SAS 9.4 statistical software (SAS Institute Inc, Cary, NC).

\section{RESULTS}

The cohort of 1048 patients consisted of $624 \mathrm{MV}$ repairs and 424 MVRs. Mean ages were $64.1 \pm 13.2$ years and $65.9 \pm 14.9$ years, and female patients comprised $40 \%$ and $52 \%$ of the MV repair and MVR cohorts, respectively. As expected, the MVR cohort harbored comorbidities more frequently, including preoperative dialysis need, lung disease, history of stroke, and previous sternotomy. Elective

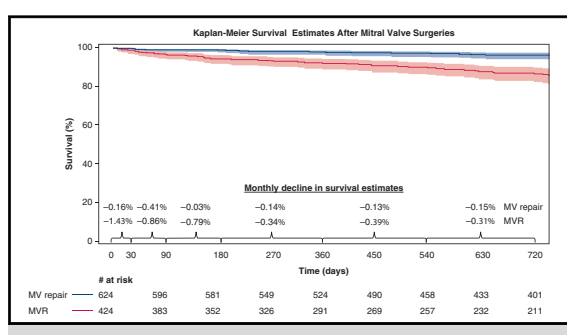

Survival estimate demonstrating persistent risk of death after mitral valve operations.

\section{Central Message}

After mitral valve operations, the risk of postoperative death persists as long as 90 days after mitral valve repair and beyond 90 days after mitral valve replacement.

See Commentaries on pages e175, e177, and e 179 .

surgery comprised $77 \%$ of the $\mathrm{MV}$ repair cohort and $58 \%$ of the MVR cohort. Isolated MV surgery comprised $64.7 \%$ of the MV repair cohort and $50.0 \%$ of the MVR cohort (Table 1).

In-hospital mortality was $0 \%$ for both cohorts. Thirtyday mortality occurred in 1 patient $(0.2 \%)$ and 6 patients $(1.4 \%)$ in the MV repair and MVR cohorts, respectively. Survival estimates after the surgery were $99.0 \%$ and $97.0 \%$ at 90 days, $98.9 \%$ and $94.5 \%$ at 180 days, and $98.0 \%$ and $92.2 \%$ at 365 days in the MV repair and MVR cohorts, respectively. Follow-up at 365 days was complete for $87 \%$ of the cohort. The mean monthly rate of decrease in Kaplan-Meier survival estimates at 0 to 30 days, 31 to 90 days, 91 to 180 days, 181 to 360 days, 361 to 540 days, and 540 to 720 days were $-0.16 \%$, $-0.41 \%,-0.03 \%,-0.14 \%,-0.13 \%$, and $-0.15 \%$ at the respective times for $\mathrm{MV}$ repair, and $-1.43 \%$, $-0.86 \%,-0.79 \%,-0.34 \%,-0.39 \%$, and $-0.31 \%$ at the respective times for MVR (Figure 1), suggesting that the elevated risk of death persisted beyond 30 days after MV surgeries and then stabilized after 90 days for MV repair but continued to persist to 180 days for MVR.

\section{DISCUSSION}

This study suggests that even after favorable inhospital and 30-day survivals after MV surgery, risk of 
TABLE 1. Patient and operative characteristics and outcomes

\begin{tabular}{|c|c|c|c|c|c|}
\hline Variable & \multicolumn{2}{|c|}{ MV repair $(N=624) \%$} & \multicolumn{2}{|c|}{$\operatorname{MVR}(N=424) \%$} & $P$ value \\
\hline Age (y; median and IQR) & 65 & $55-74$ & 68 & $57-77$ & .006 \\
\hline Female (No. and \%) & 249 & 39.9 & 220 & 51.9 & .0001 \\
\hline \multicolumn{6}{|l|}{ Diabetes (No. and \%) } \\
\hline Type 2 & 66 & 4.1 & 72 & 1.4 & $<.0001$ \\
\hline Type 1 & 20 & 1.3 & 41 & 0.8 & \\
\hline Preoperative dialysis (No. and \%) & 5 & 0.8 & 31 & 7.3 & $<.0001$ \\
\hline History of endocarditis (No. and \%) & 42 & 6.7 & 83 & 19.6 & $<.0001$ \\
\hline Chronic lung disease (No. and \%) & 23 & 3.7 & 29 & 6.8 & .021 \\
\hline Peripheral vascular disease (No. and \%) & 40 & 6.4 & 54 & 12.7 & .0004 \\
\hline History of stroke (No. and \%) & 37 & 5.9 & 65 & 15.3 & $<.0001$ \\
\hline Previous MI (No. and \%) & 93 & 14.9 & 108 & 25.5 & $<.0001$ \\
\hline Redo sternotomy (No. and \%) & 26 & 4.2 & 113 & 26.7 & $<.0001$ \\
\hline History of CABG (No. and \%) & 8 & 1.3 & 33 & 7.8 & $<.0001$ \\
\hline Congestive heart failure (No. and \%) & 220 & 35.3 & 264 & 62.3 & $<.0001$ \\
\hline \multicolumn{6}{|l|}{ Operative } \\
\hline \multicolumn{6}{|l|}{ Case status (No. and \%) } \\
\hline Elective & 482 & 77.2 & 246 & 58.0 & $<.0001$ \\
\hline Urgent & 139 & 22.3 & 165 & 38.9 & \\
\hline Emergency & 3 & 0.5 & 13 & 3.1 & \\
\hline Crossclamp time (min; median and IQR) & 90 & $73-112$ & 102 & $78-130$ & $<.0001$ \\
\hline Myxomatous disease (No. and \%) & 520 & 83.4 & 60 & 14.2 & $<.0001$ \\
\hline Concomitant CABG (No. and \%) & 120 & 19.2 & 82 & 19.3 & .97 \\
\hline Myxomatous MV* (No. and \%) & 65 & 54.2 & 13 & 15.8 & $<.0001$ \\
\hline Concomitant TV repair (No. and \%) & 47 & 7.5 & 61 & 14.4 & .0003 \\
\hline Concomitant maze (No. and \%) & 129 & 20.7 & 91 & 21.5 & .83 \\
\hline Isolated MV (No. and \%) & 404 & 64.7 & 212 & 50.0 & $<.0001$ \\
\hline \multicolumn{6}{|l|}{ Postoperative } \\
\hline In-hospital mortality (No. and \%) & 0 & 0.0 & 0 & 0.0 & - \\
\hline 30-day mortality (No. and \%) & 1 & 0.2 & 6 & 1.4 & .0144 \\
\hline Stroke (No. and \%) & 2 & 0.3 & 13 & 3.1 & .0002 \\
\hline
\end{tabular}

$\overline{M V}$, Mitral valve; $M V R$, mitral valve replacement; $I Q R$, interquartile range; $M I$, myocardial infarction; $C A B G$, coronary artery bypass grafting; $T V$, tricuspid valve. *Denominator is concomitant coronary artery bypass grafting.

mortality may persist beyond 30 days but declines substantially within 1 year. Likely owing to the difference in patient characteristics, risk of death appears to have stabilized earlier (by 90 days) in the MV repair cohort whereas it persisted beyond 90 days in the MVR cohort. This work informs current efforts in improving the postacute phase of care by defining the time frames and magnitude of the persistent risk of death after MV operations.

Most existing cardiac surgical outcome measures are limited to 30 days after the index operation and the attributions are to the hospital and the surgeon. Beyond this time frame, no measure mechanism exists, and there is a lack of systematic incentive for the health care system to monitor or improve outcomes beyond 30 days. Central question previous to investing in such effort is whether the elevated risk of death after the operation persists beyond the 30-day period. ${ }^{4}$ Our data may suggest that the elevated risk does persist, and the duration of elevated risk of death differs by the risk profile of patients, as related to case characteristics. MV operations presented an ideal contrast of risk profiles between repair and replacement cohorts to study this, with isolated repair associated with the national 30-day mortality of $1 \%$ and replacement associated with a 5\% mortality. ${ }^{5}$ Identification of cohorts likely to face persistent risk of death after MV operations may guide targeted monitoring and intervention to mitigate this postoperative hazard. Evolution of percutaneous technology with shifting patient risk profile also warrants ongoing characterization of this phenomenon.

Limitations of the study include the single-center setting, which potentially limits the generalizability, and the limited follow-up beyond 12 months. Assessment of the continuous change in the risk of death was limited by the relatively low event incidences. 
Kaplan-Meier Survival Estimates After Mitral Valve Surgeries

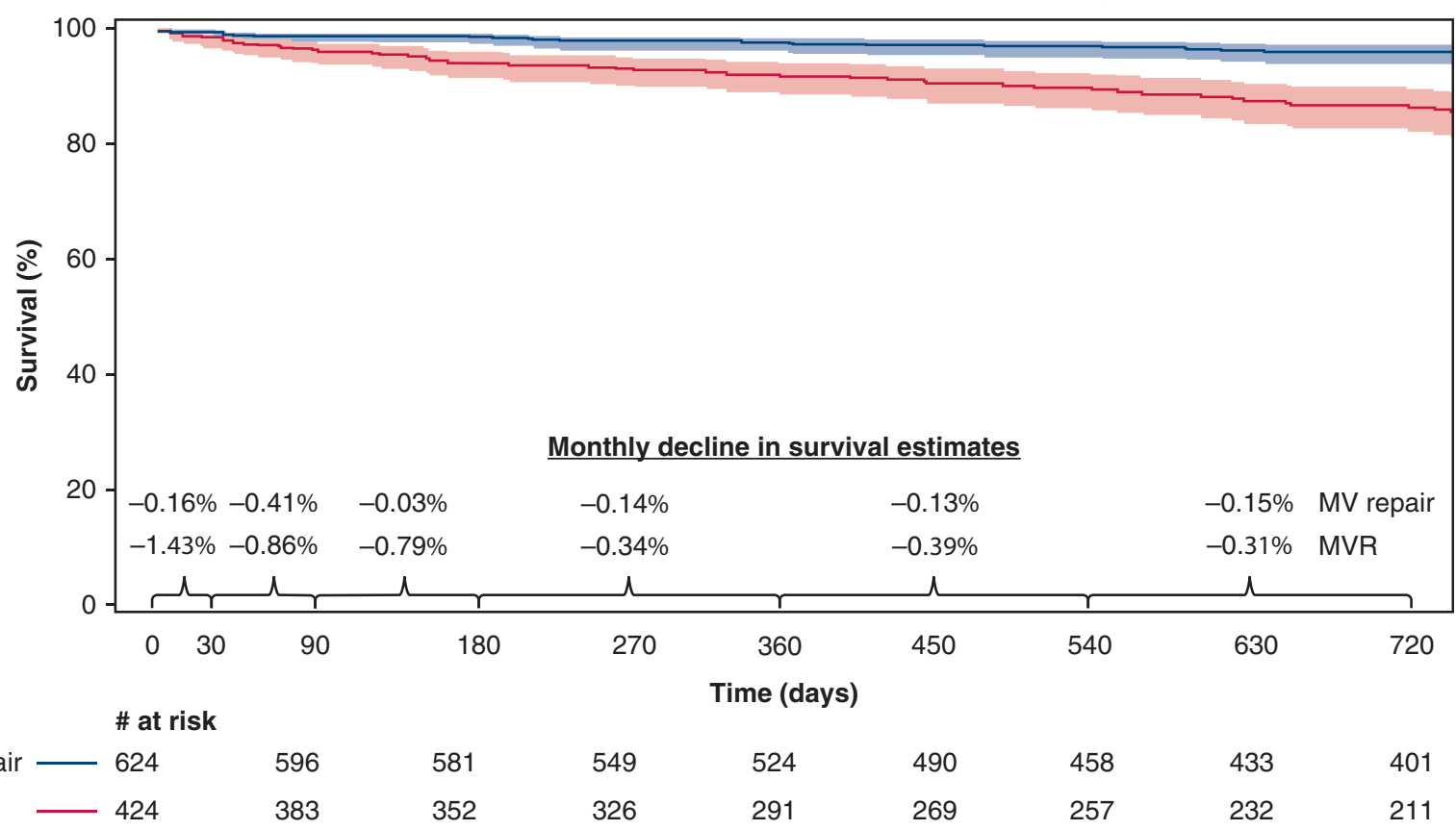

FIGURE 1. One-year survival estimate after mitral valve $(M V)$ repair and mitral valve replacement $(M V R)$. Figure shows Kaplan-Meier survival estimate during the 2 years after mitral valve operations. The blue line and red values correspond to mitral valve repair, and the red line and red values correspond to mitral valve replacement. Changes in the monthly risk of death are displayed as percentage values, with stabilization after 90 days in mitral valve repair cohort, whereas risk continued to change during 2 years in the mitral valve replacement cohort.

\section{References}

1. Shahian DM, O’Brien SM, Sheng S, Grover FL, Mayer JE, Jacobs JP, et al. Predictors of long-term survival after coronary artery bypass grafting surgery: results from the Society of Thoracic Surgeons Adult Cardiac Surgery Database (the ASCERT study). Circulation. 2012;125:1491-500.

2. Khera R, Dharmarajan K, Wang Y, Lin Z, Bernheim SM, Wang Y, et al. Association of the Hospital Readmissions Reduction Program with mortality during and after hospitalization for acute myocardial infarction, heart failure, and pneumonia. JAMA Netw Open. 2018;1:e182777.
3. Liao JM, Navathe AS, Press MJ. Medicare's approach to paying for services that promote coordinated care. JAMA. 2019;321:147-8.

4. Siregar S, Groenwold RH, de Mol BA, Speekenbrink RG, Versteegh MI, Brandon Bravo Bruinsma GJ, et al. Evaluation of cardiac surgery mortality rates 30-day mortality or longer follow-up? Eur J Cardiothorac Surg. 2013;44 875-83.

5. Crestanello JA. Quality of mitral valve surgery at the United States Department of Veterans Affairs. J Thorac Cardiovasc Surg. 2018;155: 80-1. 